\title{
INFLUÊNCIA DA PENETRAÇÃO DE INSTRUMENTOS, EMPREGADOS NA CONDENSAÇÃO LATERAL ATIVA, NA QUALIDADE DA OBTURAÇÃO DE CANAIS RADICULARES
}

\author{
INFLUENCE OF THE PENETRATION OF INSTRUMENTS, USED \\ IN THE ACTIVE LATERAL CONDENSATION, IN THE QUALITY \\ OF ROOT CANAL FILLING
}

Bethânia Camargo PINHEIRO

Especialista em Endodontia pelo Hospital de Reabilitação de Anomalias Craniofaciais, Universidade de São Paulo (HRAC/USP),Bauru-SP.

Alexandre Silva BRAMANTE

Professor Doutor em Endodontia pela Faculdade de Odontologia de Bauru, Universidade de São Paulo (FOB/USP), Bauru-SP.

Renata Pardini HUSSNE

Endodontista do HRAC/USP, Bauru-SP e Mestranda em Endodontia pela Faculdade de Odontologia da Universidade Estadual Paulista "Júlio de Mesquita Filho" (UNESP), Araraquara-SP.

\begin{abstract}
$\bar{O}$ bjetivo: comparar a qualidade da obturação e a diferença de profundidade de penetração, no interior do canal radicular, dos espaçadores digitais (Finger Spreader), limas tipo Kerr, Flexofile e Nitiflex, quando utilizados como espaçadores, durante a obturação pela Técnica da Condensação Lateral Ativa. Material e Métodos: foram utilizados 40 dentes molares permanentes humanos, extraídos, instrumentados pela Técnica de Goerig Modificada e divididos em quatro grupos. No grupo I foi utilizado o espaçador digital C; no grupo II, lima tipo Kerr; no grupo III, lima Flexofile e, no grupo IV, lima Nitiflex, todos com diâmetro $\left(\mathrm{D}_{1}\right)$ ou diâmetro da ponta correspondente ao diâmetro de uma lima $30(0,30 \mathrm{~mm})$, para abertura de espaço para colocação dos cones secundários, durante a Técnica da Condensação Lateral Ativa. Avaliou-se e submeteu-se à análise estatística (Kruskal-Wallis e teste de Dunn) a qualidade radiográfica da obturação e a profundidade de penetração dos instrumentos. Resultados e Conclusão: quanto à análise radiográfica da qualidade da obturação, o grupo II foi o que apresentou o melhor resultado, seguido dos grupos I, IV e III, respectivamente. Em relação à profundidade de penetração dos instrumentos, o grupo IV aproximou-se mais da distância desejada, ou seja, 1 milímetro aquém do comprimento de trabalho, seguido, respectivamente, dos grupos II, III e I.
\end{abstract}

UNITERMOS: Obturação do canal radicular; Instrumentos odontológicos; Espaçadores.

\section{INTRODUÇÃO}

A obturação do canal radicular consiste no preenchimento do canal radicular por materiais física e biologicamente compatíveis, visando promover seu selamento o mais hermeticamente possível. Seu sucesso depende de fases preparatórias, da forma como é realizada e dos materiais empregados para a mesma 3. Para tanto, esses materiais devem apresentar, preferentemente, alguns requisitos como, ser inertes, anti-sépticos e estimular a reparação dos tecidos periapicais, após o tratamento endodôntico ${ }^{10,12}$.

A obturação dos canais radiculares até a união cemento-dentina-canal, ou suas proximidades, é um procedimento de grande importância, pois a permanência de um espaço vazio, após uma obturação deficiente, poderia ser comprometedora para os bons resultados que se espera obter do tratamento. Isto 
porque, nos casos de lesões periapicais, poderia haver drenagem de exsudatos para o interior da porção não obturada, aí se estagnando. Com a degradação desses elementos, ocorre a produção de substâncias tóxicas irritantes aos tecidos periapicais. Estes, sofrendo esta agressão tóxica, se inflamariam mais intensamente e formariam mais exsudato. Desta forma ocorreria um verdadeiro círculo vicioso da inflamação ${ }^{10,12}$.

Dos materiais disponíveis no mercado, o cone de guta-percha, em associação com o cimento obturador, é o material consagrado para a obturação dos canais radiculares ${ }^{14,15}$.

No que diz respeito às técnicas empregadas (das quais se registra um grande número, ora adequadas ao material utilizado, ora de acordo com as condições do canal em tratamento), todas elas têm um objetivo comum: reunir qualidade com praticidade. Dentre todas, a mais utilizada universalmente é a Técnica da Condensação Lateral, devido à sua simplicidade, baixo custo, boa compactação ao nível do terço apical e ótima qualidade final 4, 11,15. Esta técnica, aparentemente proposta por Callahan ${ }^{15} \mathrm{em} 1914$, refere-se à colocação sucessiva de cones secundários lateralmente a um cone principal bem adaptado e cimentado no canal. $\mathrm{O}$ espaço para os cones secundários é normalmente criado pela ação de um instrumento ${ }^{7}$.

A Técnica da Condensação Lateral é relativamente fácil de ser executada e oferece uma colocação controlada do material obturador, diminuindo a chance de sobreobturação e, portanto, reduzindo o desconforto do paciente. A obturação obtida proporciona estabilidade dimensional e, dependendo da qualidade da obturação e do cimento empregado, pode ser facilmente removida, se necessário ${ }^{13}$.

Alguns instrumentos podem ser empregados para fazer a Condensação Lateral Ativa, como espaçadores ou a lima tipo Kerr número 30. Os espaçadores são instrumentos metálicos destinados a abrir espaço para colocação dos cones secundários durante a obturação. São comercializados com cabos manuais e digitais e em diferentes calibres ${ }^{16}$. Apresentam uma forma cônica lisa, com ponta aguda ou romba, são fabricados em aço inoxidável ou níquel-titânio, sendo estes últimos mais flexíveis e, por isso, recomendados para a obturação de canais curvos ${ }^{2,8}$. A grande maioria dos autores prefere os espaçadores digitais aos digitopalmares, por acreditarem que com eles é possível obter um melhor controle das manobras de condensação ${ }^{15}$.

$\mathrm{O}$ instrumento deve penetrar ao lado do cone principal, forçando-o de encontro a uma das paredes do canal. O espaçador não pode interferir na adaptação apical do cone principal, devendo, portanto, penetrar alguns milímetros aquém do Comprimento Real de Trabalho 1,2,10,11. Como os cones de guta-percha possuem certa elasticidade, ao remover-se o espaçador tem-se aberto um espaço que será preenchido com a colocação de um cone secundário envolto em cimento. Repetimos o ato operatório da condensação lateral, colocando mais cones secundários, até que o canal fique bem preenchido ${ }^{10}$.

A utilização de lima tipo Kerr, número 30, foi proposta por Bramante, et $\mathrm{al}^{3}$., no ano de 1972 , em substituição aos espaçadores convencionais. Esta técnica apresenta a vantagem de que a lima tipo Kerr de número 30, penetra com maior facilidade em canais atresiados, favorecida pelo guia de penetração e dos movimentos que ela permite, facilitando a condensação e a criação de espaço semelhante ao cone secundário. Outra vantagem está que o cimento pode ser levado e deixado no espaço aberto pelo instrumento sem interferir no assentamento dos cones colocados anteriormente desde que se aplique uma rotação antihorária contínua para sua remoção do canal radicular 3

A condensação lateral, realizada sob diferentes condições, pode resultar em uma qualidade diferente de obturação. Existe uma variedade de modelos de espaçadores e a maioria dos operadores tem tendências subjetivas na escolha desses espaçadores, supondo que a qualidade da obturação seja a mesma, com diferentes instrumentos ${ }^{14}$.

\section{OBJETIVOS}

Os objetivos do presente trabalho são os de avaliar Técnica de Condensação Lateral, em canais curvos e retos, comparando quatro tipos de instrumentos (espaçador digital, lima tipo Kerr, Flexofile e Nitiflex), quanto a sua capacidade de penetração no interior do canal radicular e da qualidade final da obturação. Verificar se, com a utilização de instrumentos flexíveis, como as limas Flexofile e Nitiflex, é possível obter uma obturação satisfatória em canais curvos e retos, utilizando a Técnica da Condensação Lateral Ativa.

\section{MATERIAL E MÉTODOS}

Foram utilizados 40 dentes molares permanentes humanos extraídos, sendo 20 superiores e 20 inferiores, mantidos em $100 \%$ de umidade até o momento da instrumentação. Os dentes foram retirados do Formol - solução 37\% (Rioquímica), lavados em água corrente por 10 minutos e radiografados. A 
abertura coronária foi realizada com broca esférica diamantada de número 1014 (KG Sorensen Ind. e Com. Ltda. São Paulo) e complementada com broca diamantada de extremidade inativa, de número 2082 ou 3083 (KG Sorensen). Após a localização dos canais, a embocadura dos mesmos foi dilatada com brocas de Batt de números 12 e 14 (Les Fils D’Auguste Maillefer S. A. - Suíça), para facilitar o acesso ao ápice. Todos os canais dos molares foram instrumentados pela Técnica de Goerig Modificada ${ }^{10}$, empregando lima tipo Kerr (Les Fils D’Auguste Maillefer S. A. - Suíça), tipo Hedströen (Les Fils D'Auguste Maillefer S. A. Suíça) e broca de Gates Glidden de números 2 e 3 (Les Fils D’Auguste Maillefer S. A. - Suíça). O "Batente Apical"9 foi confeccionado com uma lima tipo Kerr de número 25 ou 30 para canais curvos (mesiais dos molares inferiores e vestibulares dos molares superiores) e de número 40 para canais retos (distal dos molares inferiores e palatino dos molares superiores), a $1 \mathrm{~mm}$ aquém do ápice, confirmado por uma radiografia de odontometria. A cada troca de limas, foi efetuada uma copiosa irrigação com hipoclorito de sódio a 1,0\% (Lab. Lepetit S.A.), sendo esta solução neutralizada por uma irrigação final com soro fisiológico (J.P. Indústria Farmacêutica S.A.). Os canais foram secos com cone de papel absorvente (DiaDent - Diamont Dental Industrial Co. Ltda.) e preenchidos com uma solução de EDTA (Biodinâmica Química e Farmacêutica Ltda.) por 3 minutos ${ }^{9}$. Novamente os canais foram irrigados com soro fisiológico e secos por meio de aspiração absoluta complementada com pontas absorventes.

A seguir, os cones principais de guta-percha (Tanari - Tanariman Industrial Ltda.), selecionados de acordo com o "Instrumento Memória"9 de cada canal radicular, foram introduzidos em toda a extensão instrumentada, permanecendo travados à penetração no sentido apical e tendo sido efetuada nova tomada radiográfica. A obturação foi realizada por meio da Técnica Biológica Controlada ${ }^{9}$, utilizando cimento Sealer 26 (Dentsply), manipulado de acordo com as instruções do fabricante.

Os dentes foram divididos em 4 grupos, com 5 molares superiores e 5 inferiores cada, de acordo com os instrumentos utilizados na Condensação Lateral Ativa. No grupo I, foi utilizado o espaçador digital C (Les Fils D’Auguste Maillefer S. A. - Suíça), de 21 $\mathrm{mm}$, por apresentar diâmetro compatível com a lima tipo Kerr número 30 . O espaçador penetrou ao lado do cone principal, forçando-o de encontro às paredes do canal radicular. Foram utilizados a lima tipo Kerr número 30 (grupo II), lima Flexofile (grupo III) e lima Nitiflex (grupo IV). Estes instrumentos penetraram entre o cone principal e uma das paredes do canal radicular, por meio de pressão em direção ao ápice. Foram aplicados ao instrumento movimentos de cateterismo, alternadamente nos sentidos horário e anti-horário, dando $1 / 4$ a $1 / 2$ volta em cada um. Em seguida, foi removido o instrumento, aplicando-se-lhe movimentos de rotação anti-horária, como que desparafusando-o. Em todos os grupos após a remoção do instrumento, o espaço aberto foi preenchido com um cone secundário B7 (Tanari- Tanariman Industrial Ltda.) envolto em cimento e a profundidade de penetração do espaçador foi medida com auxílio de uma régua milimetrada.

Quando o nível de penetração do instrumento se localizou nas proximidades da embocadura do canal, considerou-se satisfatória a condensação lateral e o dente foi radiografado.

$\mathrm{O}$ excesso de material obturador que ficou na câmara pulpar foi removido com cureta aquecida e a condensação vertical realizada com os condensadores verticais (Irmãos Aronson - São Paulo, Brasil). A limpeza da cavidade foi feita com éter, uma bolinha de algodão foi colocada na embocadura dos canais e as aberturas coronárias foram seladas com cimento provisório, Cimpat (Septodont - Specialites Septodent, Paris - França - DFL - Rio de Janeiro, Brasil), com 4 mm de espessura.

As radiografias finais foram avaliadas por meio de um negatoscópio e de acordo com a qualidade da obturação, atribuiu-se escores de 1 a 3 para os seguintes parâmetros: 1 , excelente; 2 , satisfatória e 3, insatisfatória.

Foi medida a diferença entre o comprimento de trabalho e o comprimento de penetração do instrumento utilizado para abrir espaço na massa obturadora.

Os dados obtidos da qualidade da obturação e profundidade de penetração do instrumento foram avaliados por meio da análise estatística de KruskalWallis e teste de Dunn.

\section{RESULTADOS}

Quando se comparou a qualidade da obturação, o teste de Kruskal-Wallis mostrou diferença estatisticamente significante entre os grupos analisados $(\mathrm{H}=36,29 ; \mathrm{P}=<0,0001)$, e os dados encontram-se na Tabela 1. Para as comparações múltiplas aplicou-se o teste de Dunn e os resultados são demonstrados na Tabela 2. Na comparação entre os grupos, pode-se verificar que não houve diferença estatisticamente significante apenas entre os grupos I e II e os grupos 
III e IV.

Em relação à profundidade de penetração dos instrumentos utilizados, a média da diferença entre o comprimento de trabalho e a penetração do espaçador em milímetros e o desvio padrão estão representados na Tabela 3.

\section{DISCUSSÃO}

O sucesso da terapia endodôntica está relacionado com a correta execução de cada uma de suas fases, desde o diagnóstico até a obturação e proservação. Torna-se clara, então, a importância da fase de obturação, tendo em vista que um dos objetivos da terapia endodôntica é o preenchimento do canal em forma tridimensional, com a finalidade de se obter um selamento hermético em toda sua extensão e diâmetro ${ }^{11}$.

Embora os estudos experimentais in vitro não possam reproduzir com exatidão as condições clínicas, a melhor maneira de testar a eficácia de técnicas obturadoras é transportar os resultados experimentalmente obtidos para aquelas condições e fazer uma avaliação a longo prazo.

A Técnica da Condensação Lateral Ativa é mais comumente realizada por espaçadores digitais (Finger Spreader $)^{5,10,15}$. Esses espaçadores são hastes metálicas cilíndrico-cônicas lisas, de ponta romba, que possuem um pequeno cabo digital. São de grande utilidade durante a obturação dos canais pois, estando o cone principal em posição, abrem espaço para a colocação de cones auxiliares, permitindo, assim, um bom preenchimento do canal radicular ${ }^{10}$. A Técnica da Condensação Lateral Ativa proposta por Bramante ${ }^{3}$ et al., com limas tipo Kerr, não é ainda difundida entre os autores estrangeiros nem mesmo entre muitos brasileiros; todavia, os trabalhos publicados que a envolvem permitem concluir ser essa uma técnica ideal quanto a seu fácil manuseio e sua efetividade de selamento marginal ${ }^{4}$.

Cohen, Burns ${ }^{5}$ citam que Allisson, Michelich, Walton" observaram, in vitro, que "o grupo no qual a extremidade do espaçador poderia ser inserida a $1 \mathrm{~mm}$ do comprimento preparado apresentava uma microinfiltração consideravelmente menor do que aquela apresentada no grupo em que era grande a distância entre a extremidade do espaçador e o comprimento preparado". Berry, et $\mathrm{al}^{2}$. concluíram em seu trabalho que espaçadores mais flexíveis, construídos com liga de níquel-titânio, têm maior habilidade do que os espaçadores de aço inoxidável em atingir o comprimento de penetração desejado em canais curvos.

Deve-se procurar melhorar essa condição usando espaçadores que possam chegar mais próximo do Comprimento de Trabalho. Indubitavelmente, as curvaturas afetam a qualidade da obturação. Concordase então com Gani, et $a^{6}$. quanto ao fato de que o instrumento deveria ser "testado" no canal preparado antes de seu uso, para garantir um ajuste adequado a 1 mm do comprimento de trabalho.

Em relação à qualidade radiográfica da obturação (Tabela 1), nota-se que as limas tipo Kerr obtiveram a maior porcentagem de escores 1 (excelente), ou seja, $81,82 \%$, seguido do grupo I $(61,29 \%)$, do grupo IV $(22,58 \%)$ e do grupo III $(20 \%)$. Isto se deve, provavelmente, ao fato de que, após a introdução de alguns cones secundários, é necessária certa pressão

TABELA 1- Qualidade radiográfica da obturação

\begin{tabular}{llll}
\hline Grupo & Escore 1 & Escore 2 & Escore 3 \\
\hline I & $61,29 \%$ & $35,48 \%$ & $3,23 \%$ \\
II & $81,82 \%$ & $12,12 \%$ & $6,06 \%$ \\
III & $20 \%$ & $50 \%$ & $30 \%$ \\
IV & $22,58 \%$ & $38,71 \%$ & $38,71 \%$ \\
\hline
\end{tabular}

TABELA 2- Teste de Dunn

\begin{tabular}{ll}
\hline Comparação & Diferença entre postos \\
\hline IV X II & $40,689^{*}$ \\
IV X I & $31,258^{*}$ \\
IV X III & 1,571 \\
III X II & $39,118^{*}$ \\
III X I & $29,687^{*}$ \\
I X II & 9,431 \\
\hline
\end{tabular}

* - Diferença estatisticamente significante $H=15,378 ; P=0,002$

TABELA 3- Médias, em milímetros, da diferença entre o comprimento de trabalho (CT) e a profundidade de penetração do instrumento $(E)$ e, os respectivos desvios padrões (D.P.)

\begin{tabular}{lll}
\hline Grupo & CT $-\mathbf{E}$ & D. P. \\
\hline I & 2,177 & 1,604 \\
II & 1,606 & 1,235 \\
III & 1,833 & 1,347 \\
IV & 1,048 & 0,373 \\
\hline
\end{tabular}


na colocação do instrumento para que seja criado novo espaço, o que é melhor conseguido com instrumentos mais rígidos, pois os mais flexíveis tendem a dobrarse quando aquela certa pressão é empregada.

Em relação à Tabela 2, quando se comparou, através do teste de Dunn, os grupos IV com III e I com II, não houve diferença estatisticamente significante $(\mathrm{H}<15,378)$, por se tratar de instrumentos com flexibilidade semelhante, porém quando foram comparados instrumentos rígidos com os mais flexíveis, como nos grupos IV com II; IV com I; III com II e III com I constataram-se diferenças estatisticamente significantes entre eles $(H>15,378)$, pois, quanto a qualidade da obturação, os instrumentos rígidos demonstraram resultados estatísticos superiores.

Em relação a profundidade de penetração dos instrumentos, quem melhor alcançou a distância de 1 $\mathrm{mm}$ do comprimento de trabalho foi a lima Nitiflex (1,04 mm em média), seguido da lima Kerr (1,60 mm), Flexofile $(1,83 \mathrm{~mm})$ e espaçador digital $(2,17 \mathrm{~mm})$. A falta de flexibilidade dos instrumentos de aço inoxidável utilizados não permitiu alcançar a distância de $1 \mathrm{~mm}$ do comprimento de trabalho em canais curvos; entretanto, a dureza desses instrumentos é preferida por alguns clínicos por permitir uma compactação mais firme. $\mathrm{O}$ cone secundário de gutapercha, devido a sua consistência elástica, não é capaz de penetrar na porção mais apical do espaço aberto por instrumentos flexíveis, ocasionando falhas na obturação.

\section{CONCLUSÃO}

Diante dos resultados obtidos e da metodologia empregada na realização deste trabalho, pode-se concluir que:

- Na análise radiográfica da qualidade da obturação, o grupo II, em que se utilizou a lima tipo Kerr, apresentou o melhor resultado, seguido do grupo I, com o espaçador digital (Finger Spreader), do IV, com a lima Nitiflex, e, por último, do grupo III, em que foi utilizada a lima Flexofile.

- Em relação à profundidade de penetração dos instrumentos, a lima Nitiflex aproximou-se mais da distância desejada, ou seja, 1 milímetro aquém do comprimento de trabalho, seguido, respectivamente, das limas Kerr, Flexofile e do espaçador digital.

- Os instrumentos flexíveis alcançaram maior profundidade de penetração no interior do canal que os instrumentos rígidos, porém muitas vezes o espaço criado não era preenchido pelo cone secundário em sua totalidade, ocasionando espaços vazios que interferiram na qualidade da obturação.

Baseado nas conclusões anteriores, sugerimos que a Condensação Lateral Ativa continue a ser realizada com instrumentos mais rígidos, facilitando a penetração dos cones secundários em todo o espaço anteriormente criado.

\section{ABSTRACT}

Purpose: the purpose of this study was to compare the quality of the obturation and the difference of penetration depth inside the root canal of the instruments Finger Spreader, file type Kerr, Flexofile and Nitiflex when used as spreaders during the filling by Active Lateral Condensation Technique. Material and Method: 40 extracted permanent human teeth were instrumented by the Modified Goerig Technique and divided in four groups. In the group I the finger spreader was used; in the group II, files type Kerr was used; in the group III, files Flexofile was used and, in the group IV, files Nitiflex, all number 30, for space opening for placement of the secondary cones, during the Technique of Active Lateral Condensation. The radiographic quality of the obturation and the depth of penetration of the instruments was evaluated and analysed statisticaly (Kruskal-Wallis and Dunn's Test). Results and Conclusion: with relationship to the radiograph analysis of the quality of the filling, the group II was what it presented the best result, followed by the groups I, IV and III, respectively. In relation to the depth of penetration of the instruments, the group IV approached more the desired distance, or, 1 millimeter less than the length of the work, followed, respectively, by groups II, III and I.

UNITERMS: Root canal obturation; Dental instruments; Finger spreader.

\section{REFERÊNCIAS BIBLIOGRÁFICAS}

1- Allison DA, Michelich RJ, Walton RE. The influence of master cone adaptation on the quality of the apical seal. J Endod 1981; $7: 61-5$.

2- Berry KA, Loushine RJ, Primack PD, Runyan DA. Nickeltitanium versus stainless-steel finger spreaders in curved canal. J Endod 1998; 22:752-4.

3- Bramante CM, Berbert A, Piccino AC. Técnica de condensação lateral para obturações de canais radiculares de pequenos diâmetros, com cones de guta-percha. Estomatol Cult 1972; 6:702 
4- Bramante CM, Berbert A, Tanomaru Filho M, Moraes IG. Estudo comparativo de algumas técnicas de obturação de canais radiculares. Rev Bras Odontol 1989; 46:26-35.

5- Cohen S, Burns RC. Caminhos da polpa. 6a. ed. Rio de Janeiro: Guanabara Koogan; 1997.

6- Gani O, Visvisian C, Caso C. Quality of apical seal in curved canals using three types of spreaders. J Endod 2000; 26:581-5.

7- Glickman GN, Gutman JL. Contemporary perspectives on canal obturation. Dent Clin North Am 1992; 36:327-41.

8- Joyce AP, Loushine RJ, West LA, Runyan DA, Cameron SM. Photoelastic comparison of stress induced by using stainless-steel versus nickel-titanium spreaders in vitro. J Endod 1998; 22:7145 .

9- Leonardo MR, Esberard RM, Bonetti Filho I, Leonardo RT, Tanomaru Filho M. Tratamento de canais radiculares: atualidades técnicas. São Paulo: Premier; 1996.

10- Leonardo MR, Leal JM. Endodontia: tratamento de canais radiculares. $3^{\text {a }}$.ed. São Paulo: Panamericana; 1998.

11- Luccy CT, Weller RN, Kulild JC. An evaluation of the apical seal produced by lateral and warm lateral condensation techniques. J Endod 1990; 16:170-2.

12- Paiva JG, Antoniazzi JH. Endodontia: bases para a prática clínica. 2a.ed. São Paulo: Artes Médicas; 1988.

13- Sakkal S, Weine FS, Lemian L. Lateral condensation: inside view. Compendium 1991; 12:796-800.

14- Simons J, Ibanez B, Friedman S, Trope M. Leakage after lateral condensation with finger spreaders and D-11-T spreaders. J Endod $1991 ; 17: 101-4$.

15- Soares IJ. Endodontia: técnicas e fundamentos. Porto Alegre: Artes Médicas; 2001.

16- VanGheluwe J, Wilcox LR. Lateral condensation of small, curved root canals: comparision of two types of accessory cones. J Endod 1996; 22:540-2.

Recebido para publicação em: 26/02/2003

Aceito após reformulações: 15/05/2003

Bethânia Camargo Pinheiro

Rua Garcia Braga, 126 - Centro

São Pedro do Turvo - SPFone: (14) 33771190

e-mail: bethaniacp@uol.com.br 